\title{
Multigate Mesh Routing for Smart Grid Last Mile Communications
}

\author{
Hamid Gharavi and Bin $\mathrm{Hu}$ \\ Advanced Network Technologies \\ National Institute of Standards and Technology \\ Gaithersburg, USA \\ Emails: [gharavi, bhu]@ nist.gov
}

\begin{abstract}
One of the most important issues in Smart Grid is to provide consumers with the knowledge of their energy usage, as well as allowing the utility to monitor and control the electric system components with real time status. This would require the design of a network architecture that is capable of providing secure and reliable two-way communication from meters to other Smart Grid domains. While networking technologies and systems have been greatly enhanced, in wireless communication environments the smart grid faces new challenges in terms of reliability. In this paper we are mainly concerned with assessing the performance of wireless mesh networks for the last mile communication. We consider a tree-based mesh routing scheme based on a multigate mesh network architecture. The multigate mesh routing scheme is based on a flexible mesh network architecture that expands on the hybrid tree routing of the IEEE 802.11s. The network is specifically designed to operate in a multi gateway structure in order to meet the smart grid requirements in terms of reliability, self-healing, and throughput performance. To further exploit the multigate network structure, a timer-based multipath routing diversity scheme is proposed in order to enhance the network reliability. The network is then used to measure the performance in terms of throughput and reliability.
\end{abstract}

Keywords-Smart Grid, wireless mesh networks, routing protocols, WLAN, IEEE 802.11 s

\section{INTRODUCTION}

The Smart Grid is comprised of many networks (domains) with various boundaries that have to be interconnected to provide end-to-end services. The challenge is to design multiple network architectures that can meet the interoperability requirements for inter and intra domain communications. Inter and Intra domain communications can be found in the NIST conceptional reference model [1]. Such networks, as defined in the Energy Independence and Security Act of 2007, have to provide a secure and reliable two-way communications system. One of the most important issues for providing two-way communications is handling the so-called last mile communication, which covers the connectivity from the meters to the Advanced Metering Infrastructure (AMI) headend. The design of such a network, which is also referred to as the Neighborhood Area Network (NAN), depends not only on the application layer requirement, but also on the nature of its Medium Access Control (MAC) and physical (PHY) layer. There are a number of diverse communications technologies and standards that can be deployed for NAN. In terms of the physical links, these technologies can be broadly classified into two major categories: wireless and wired.

One example of the latter is Power Line Communication (PLC), which includes Broadband PLC (BPLC) [2], [3] and Narrowband PLC (NBPLC) standards [4]. At the same time Wireless LAN (WLAN) techniques, such as the IEEE 802.11 family standards [5] with their maturity and cost effectiveness, have been extensively deployed for wireless access and home entertainment networking. For smart grid, the main issue is how to effectively apply this technology to handle the last mile communication. Figure 1 presents an example of network architecture showing communication between meters and an AMI headend via Data Aggregator Points (DAPs) located on neighborhood distribution poles. Note that a DAP in this figure acts as a gateway between a local access point and the meters associated with it. For instance, IEEE 802.11 WLAN Access Point (AP) [5], which operates in a single hop (infrastructure mode), can be used to represent a DAP. However, to provide a large coverage area, multihop communication is vastly favored over long-range single-hop links. Indeed, the use of multihop is to combat the rapid decay of the electromagnetic received signal strength as communication distance increases. In addition, multihop communication between distributed nodes offers pathways around electromagnetic transmission obstacles that would otherwise prevent the formation of a long-range network [6].

In order to deploy IEEE 802.11 WLAN devices for network implementation using mesh topology, it would be necessary for mesh nodes to operate in distributed mode (i.e., as a router) [6]. Bear in mind that IEEE 802.11 WLAN standards can either function in an infrastructure mode (single hop) or a distrusted mode (multihop). Therefore, to form a peer-to-peer multihop mesh topology in the form shown in Fig. 1, it would be essential that mesh nodes operate in both modes

For the IEEE $802.11 \mathrm{~b}$ for instance, such an arrangement that can expand the functionality of the extended service set (ESS) was developed in [6]. As a result, a slave node (i.e., station representing a home appliance), which does not have mesh capability through its access point can send data over the mesh network. In this configuration the routing was provided at layer 3 . 
Recently, the IEEE 802.11 s group extended multihop mesh techniques to specify the functionality of a wireless distribution system that interconnects 802.11 devices [7]. An important feature of the IEEE 802.11s is that it supports frame forwarding and path selection at layer-2. For example, the routing protocol uses MAC addresses and an optional radio-aware routing metric that can support unicast, multicast, and broadcast data delivery. In order to make the difference to routing on layer 3 with IP addresses more distinct, the preferred term for routing is path selection in IEEE 802.11s. Although IEEE 802.11s modifies the MAC, it does not require any changes to the PHY layer and hence, its implementation is based on the existing PHY layer of IEEE $802.11 \mathrm{a} / \mathrm{b} / \mathrm{g} / \mathrm{n}$. In addition, it is fully compatible with higher layer protocols. IEEE $802.11 \mathrm{~s}$ also defines a default routing protocol, which is known as the Hybrid Wireless Mesh Protocol (HWMP).

In this paper however, our objective is to develop routing protocols that are specifically designed to meet the requirements of Smart Grid, which includes a high degree of reliability, self-configuring, and self-healing. For instance, there are a number of issues that need to be investigated to assess the suitability of the routing schemes for the last mile communication. In particular, the time-varying nature of traffic generated under emergency poses a significant challenge in ensuring the reliability of the network. For instance, outage management is one example where a system expects to receive power outage notifications and exchanges, which tend to increase traffic load and could consequently cause severe network congestion. More importantly, as indicated in Fig.1, metering traffic has to go through multiple DAPs in order to prevent overload at each DAP. Under these conditions an efficient routing scheme must be applied that can guarantee a fair traffic balance amongst them. Furthermore, by taking advantage of multi gateways structure, the path diversity routing scheme is proposed in order to enhance network reliability.

The paper is organized as follows: In Section II, we discuss issues related to deploying mesh network architecture using IEEE 802.11 s as its core technology. The mesh routing, which is on a hybrid tree-based routing protocol in a multiple gateway environment is presented in Section III. Section IV presents a timer-based multiple-path diversity routing scheme followed by the simulation results in section $\mathrm{V}$.

\section{MESH NETWORK ARCHITECTURE}

The architecture and design of mesh networking can play a crucial role in reliable access to/from the meters. More importantly, to avoid service disruption, routing protocols must be robust to link failures. In most cases however, covering a residential area may not terminate in a single access point, but require multiple DAPs as shown in Fig. 1. On this basis, treebased routing (TBR) protocols $[8,9]$ are deemed to be most suitable for our network. Tree-based routing is a special case of peer-to-peer network where each meter represents a leave-node that connects to the access point (DAP) via a multihop link.

In general, there are two popular tree-based routing protocols: cluster-based tree routing and hybrid tree routing. In the former, the network is divided into clusters where each cluster is represented by a cluster head. This routing protocol has been considered for Zigbee/IEEE 802.15.4 [10]. Hybrid tree-based routing protocols are generally formed as a combination of the on-demand (reactive) and proactive routing

Backhaul Network

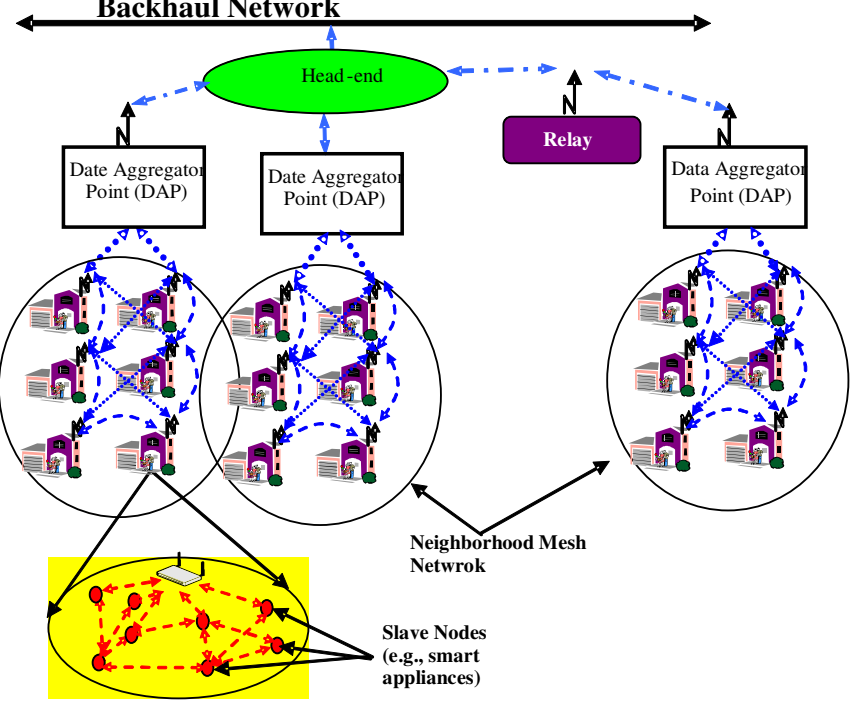

Figure.1 Mesh network architecture for the last mile smart grid.

protocols. The function of the Proactive is to maintain routing state, while the reactive aims at reducing the impact of frequent topology changes by acquiring routes on demand. An example of the hybrid tree routing is the Hybrid Wireless Mesh Protocol (HWMP). HWMP is considered as the default routing protocol for the IEEE 802.11s [7] and has been considered to implement our network. IEEE 802.11s specifies three types of nodes and for our application these nodes are defined as follows:

1) Mesh Point, representing the relay node in our model.

2) Mesh access point (MAP), which operates as the gateway of the home network to the meter and from meter toward the nearest DAP, as shown in Fig. 1. A slave node in this figure represents the in home devices (e.g., smart home appliances) and can operate as slave nodes similar to the network configuration in [6].

3) Mesh Portal point (MPP), which represents the neighborhood gateway point (DAP) and can be connected to the AMI headend via a wired backbone network or wirelessly in the absence of such a network. In either case, for our model a DAP represents a root of the tree.

As the proactive part of the routing, a DAP periodically broadcasts root announcements by increasing the sequence number each time such a message is generated. The root announcement allows proactive routing towards each meter (MAP) in the residential area. When a meter receives such a message, it catches the MAC address of the transmitting node then adds it to its list of parent nodes. It should be noted that a meter node, before re-broadcasting the root announcement message, should wait for a pre-defined time to check whether there are more of the same root announcements from other neighbor nodes. After expiration of the period the meter node, which now knows the path to the DAP as well as its MAC address, may unicast a Path Request (PREQ) through its best 
parent to the DAP acquiring a path to it. We should point out that PREQ is used by the child node (meter) to confirm the paths to its selected parent and hence validate its parent and its destination DAP. The DAP will then unicast a Path Reply (PREP) back to the meter. The tree formation process is continued by every new child (meter) by re-broadcasting the root announcement to neighboring nodes. It is important to point out that due to the static nature of the network, the root announcement plays an important role in meeting the selforganization and self-healing requirements of the smart grid. For instance, in the case of a meter malfunction, the root announcement can update the routing tree by trying to bypass the faulty nodes.

For smart grid applications where meters, DAPs, and relay nodes are predominantly stationary, the on-demand part of routing mainly deals with the effect of interference that can cause path-breakage. Note that amongst many on-demand protocols, Ad-hoc On Demand Distance Vector (AODV) [11] and Dynamic Source Routing (DSR) [12] have been perceived as the most popular and well-tested. There are a number of distinctions between the two protocols. For instance, in DSR every packet must carry the Internet protocol (IP) addresses of all the nodes along the source to the destination, whereas in AODV only the destination address is carried in each packet. Obviously, for a large network where there is the possibility of a link with a high number of hops, DSR can lead to a significant increase in the packet overhead.

\section{MUlTIPLE DAP ROUTING STRUCTURE}

According to the general architecture depicted in Fig. 1, a residential network may consist of multiple mesh sub networks where each is managed independently by its local gateway (DAP). However, due to the variable nature of the traffic, some gateways may suffer from more congestion than others. Under such conditions, nodes belonging to neighboring subnetworks cannot participate in reducing the traffic load. In order to allow collective participation in the routing, it would be advantageous to combine all the sub-networks into a larger network with multiple Gateways (DAPs) where all the meters can access any of the gateways. In addition, such an arrangement can enhance the self-healing and self-organization abilities of the network if some of the gateways and nodes become non-operational or new nodes are added to expand the network.

The first step towards achieving this is to develop flexible multigate routing in such a way that meters can have an option to select the best path to one of the gateways. With such routing flexibility together with the help of an efficient packet scheduling technique, it would then be possible to enhance network performance. Fig. 2 shows an expansion of a single DAP sub-network to a multi-DAP network. To construct a multiple routing based on the network structure shown in this figure, DAP-1, DAP-2, ... and DAP-N broadcast root announcements periodically to set up their trees. We use a randomization technique to avoid collisions. The MAC addresses of DAP-1, DAP-2, ... and DAP-N are employed as the unique identification of the corresponding routing trees. In contrast to Single DAP, a node in a Multiple DAP (MDAP)

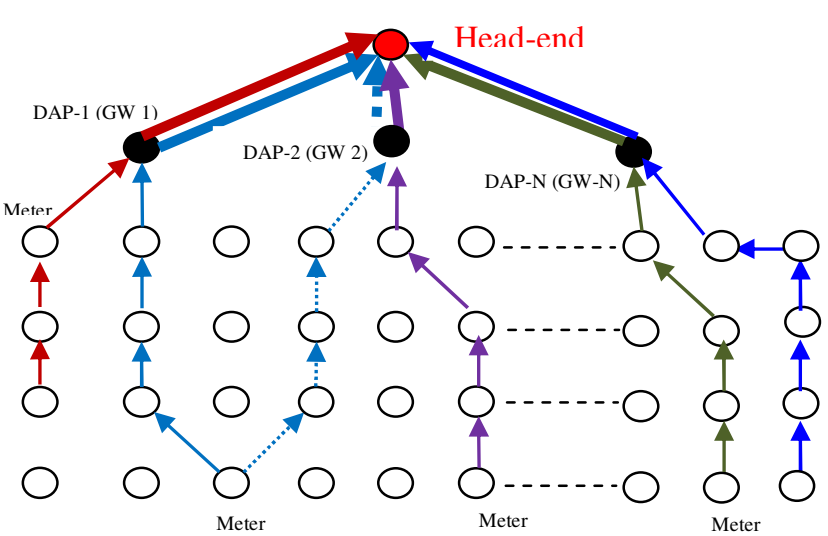

Fig. 2: An example of the Grid static wireless mesh network with an N gateway

topology has multiple entries in its Tree-Table representing a separate path to each DAP. For example, one entry represents a tree with DAP-1 as its root, which also includes its parent information, as well as the status of the tree (i.e., whether it is active or not). A mesh node (meter) when receives a (DAP) announcement, will check its tree-table to see if there is a tree with the same root announcement:

a) If not, the meter will generate a new tree in its table by using the information from the received root announcement message, where DAP and parent information, as well the treestatus are created and maintained.

$b$ ) If yes, the meter will upgrade the existing tree with the same DAP if newer or better path information is contained in the received announcement message. Otherwise it will discard this announcement message.

Meanwhile, a meter will set up a path to the new DAP in its route table if there is no route available to this DAP. It should be noted that the main objective is for every mesh node (meter) to systematically select the most suitable DAP as its gateway to the headend. To improve network reliability we further exploit this multi DAP network topology by introducing a timer-based multipath diversity routing. For instance, if the connection from one DAPS to a meter is damaged, the same service can be provided from a different route, including any of the neighboring DAPs.

\section{Reserve-PAth Multigate DiVERsity Routing}

Multiple path routing protocols for Ad Hoc networks have been extensively studied in recent years [13-17]. Generally, there are two types of multipath routing protocols: simultaneous routing and reserved-path routing. In the former approach, packets are transmitted simultaneously to the same destination, but via different paths [16], [17]. This approach could substantially increase the network load, unless methods such as multiple description coding are applied [18]. Alternatively, a reserve path approach would be the better solution in terms of bandwidth efficiency, but this would be at the expense of additional delay. 


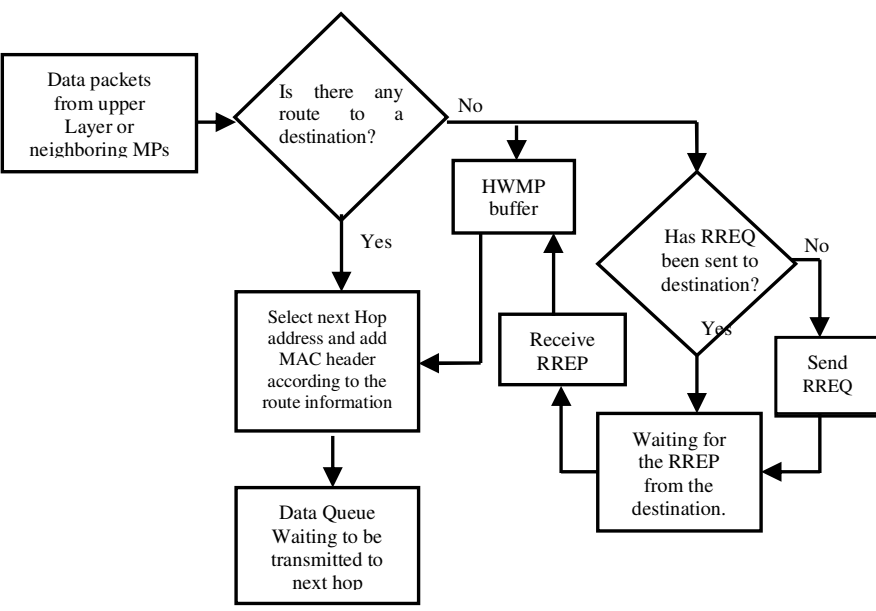

Fig. 3: Standard HWMP Routing Procedure

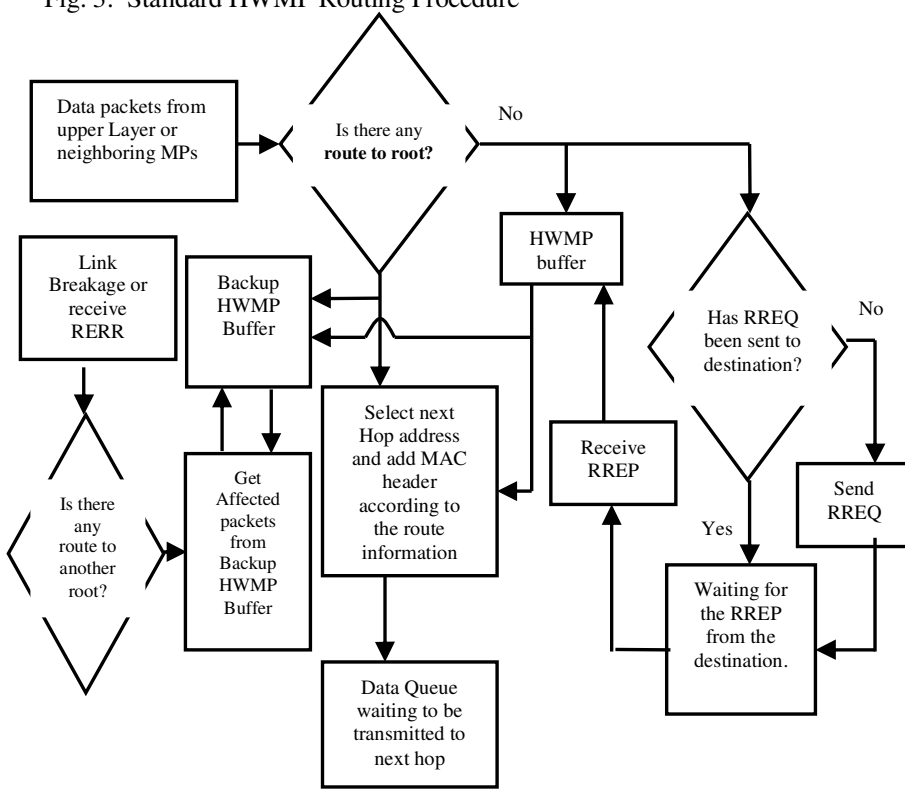

Fig. 4: Timer-based Source Reroute Multiple DAPs Routing Scheme

In this paper, we present a tree based multipath diversity routing scheme. The proposed route diversity takes advantage of the multi gateway tree-based routing scheme with the objective of managing the transmission of packets through a different path to possibly another gateway, which may suffer less from interference. For example, in the upstream link the same packet can be transmitted from a meter to the headend through a separate DAP. In the downstream link, the same packets can be transmitted from multiple neighboring DAPs to a single destination (meter).

In the case of the reserved-path diversity approach, the first best path (e.g., based on a certain path selection metric) will be selected to send a data packet. As soon as a link breakage notification is received for this path, the source node will send the packet through to the second route, if both links breaks, the new routes have to be established via on-demand routing of HWMP. For implementation, we designed a Timer-based reserved path routing scheme. To aid the description of multipath routing, Figures 3 and 4 show the standard and the Timer-based HWMP-based routing procedures. In the Timerbased reserve-path routing scheme, every meter has an additional backup HWMP buffer to store a copy of selfgenerated and already transmitted packets (see Figs. 4). Specifically, when a meter node receives data packets from its upper layer (a self generated packet) or neighboring meters (a relayed packet), it checks its route table to see if there are routes to DAP 1, DAP 2 and DAP N. If there are multiple routes, it selects the best path by adding the next hop MAC address to the MAC header field of the packets. In the case when there is only one reachable DAP, it will choose the available route to forward its packet. These packets are then inserted in the data queue, waiting to be transmitted to the next hop. Meanwhile, a copy of the self-generated packet with time stamp is backed-up in the backup buffer as shown in Fig. 4. Note that only self-generated packets are stored in the backup HWMP buffer and those received from neighboring meters are not backed-up. This is mainly to avoid multiple retransmissions.

In the case of link failure, as soon as a link breakage notification (i.e., path error message: PERR) is received, the source node will first check whether there is a route to other DAPs. If so, it retrieves the affected packets from its backup buffer and sends them to the other DAP (s). The destination address and the transmitting meter address in the PERR are used to check whether the packets in the backup buffer are affected.

If there are no routes to any DAP caused by link failures, packets will be stored in the HWMP buffer, while nodes will check if RREQ has already been sent to the originally selected DAP. If so, nodes will wait for the RREP. Otherwise, a RREQ will be sent to this DAP. When receiving RREP from the original DAP, meters get packets from the HWMP buffer and input them to the data queue after updating the MAC header. Again, a copy of self-generated packets will be put in the backup HWMP buffer for possible retransmission.

Since source meters don't know which packets have already been successfully received by any of the DAPs, they will retransmit all the affected packets in their backup HWMP buffer. In order to remove backlog packets from the backup buffer, meters will start a timer to periodically delete such packets thus reducing the possibility of retransmitting those that may have been successfully transmitted. If the timer value is too high, many of the successfully transmitted packets will be retransmitted and this can result in high overhead in the network. On the other hand, when the timer value is too low, many unsuccessfully transmitted packets will be removed from the backup - buffer and therefore cannot be retransmitted through the reserved path. A trade-off of the timer value can be achieved which helps with a good performance. Due to space limitation, we only present the best achievable performance of the Timer-based reserve-path routing scheme in this paper.

\section{Simulation RESUlts}

For simulation we first developed a residential network consisting of three wireless mesh sub-networks, according to the scenario shown in Fig. 1. In this scenario each sub-network 
is handled independently by its local gateway (DAP) before being connected to the master gateway (headend). The Hybrid Wireless Mesh Protocol (HWMP), which is the default routing protocol for the PIEEE P802.11s [7], has been used as the core routing protocol in our simulation. This protocol is then extended to form our multigate network architecture presented in section III. In this model, the routing is designed in such a way that every node will possess a separate path to each of the gateways.

We should emphasis again that, despite the static nature of the mesh network, the on-demand protocol is mainly used to cope with link failures caused by co-channel interference. The proactive part is primarily considered for the formation of the tree (e.g., self organization). In tree-based proactive routing each gateway, as the root of the tree, periodically floods the network by broadcasting a root announcement message. The period in which this message is generated depends on the nature of the application. For instance, in the case of the smart grid it should be sufficiently long to reduce excessive overheads, but short enough to handle changes in the network structure such as adding new meters or handling malfunctioning nodes (e.g., self healing). Subsequently, to enhance the reliability of the network our so-called timer-based reserve-path diversity is implemented to improve the network reliability in the presence of interference.

In the simulations the input data generated at a Variable Bit Rate (VBR), is encapsulated into fixed 512 bytes UDP packets. In the physical layer the IEEE $802.11 \mathrm{~b}$ is used and the data-rate is $2 \mathrm{Mbps}$, while gateways are assumed to have an unlimited bandwidth. The noise factor is 10.0 and the retransmission limit is 7. Three scenarios are considered in this paper. In scenario A, the network consists of three sub-networks where nodes in each are handled by their local DAP (or GW as shown in Fig. 1 ). In this scenario there are 12 meters (nodes) in each subnetwork and meters (nodes) are uniformly distributed within their coverage area.

In scenario $\mathrm{B}$, a multi-gateway network is constructed that comprises three DAPs (GWs) and 36 symmetrically distributed meters. Each DAP, as the root of the tree, broadcasts its root announcements periodically by floating the entire network. The generation of the root announcement message by each DAP has been randomized to prevent collisions. In addition, a hop-count limit of 10 has been imposed on forming a tree. This number is selected to reduce the number of paths to the far away DAPs, but at the same time making sure that every node in the network has access to at least two neighboring DAPs (via a separate path). The MAC address of the DAP is employed as the unique identification of the corresponding routing trees. In order to assess the traffic load balancing performance of our routing schemes, we also modified the structure of the network in scenario B to scenario $\mathrm{C}$, where 36 nodes are asymmetrically distributed.

Based on the above scenarios, we then evaluated the network performance in terms of overall throughput versus the input per node bit-rate. In these experiments all the nodes in the network generate data packets at VBR. Fig. 5 shows the results of the 3-subnetwroks according to scenario A, multigate bestpath scheme for Scenario B, which also includes the timer-

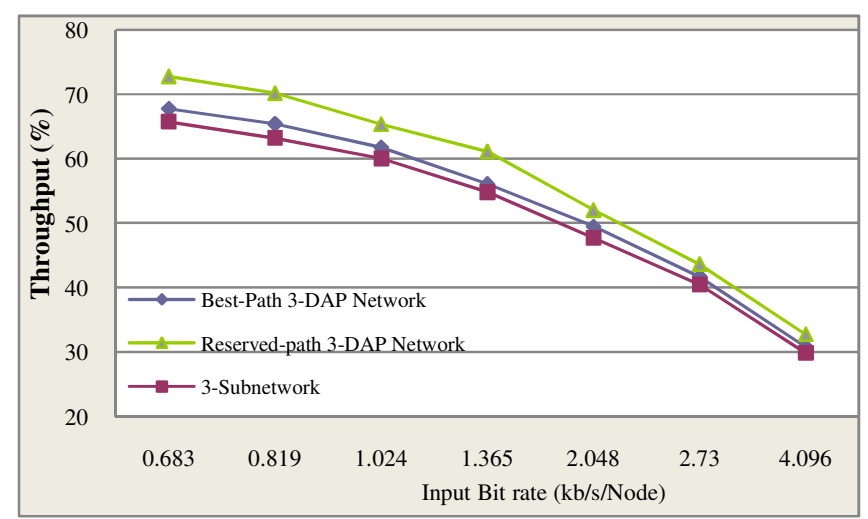

Fig. 5: Performance evalautions of the 3-Subnetwrok of scnario A, bestPath and Reserved-Path schemes in scnario B.

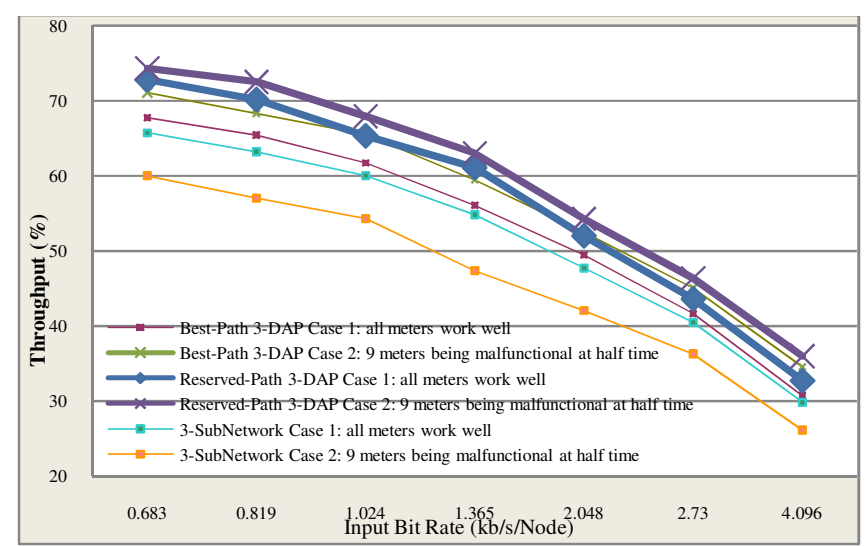

Fig. 6: Self-healing performances of in scenario B and scenario A where 9 meter nodes begins stop functioning.

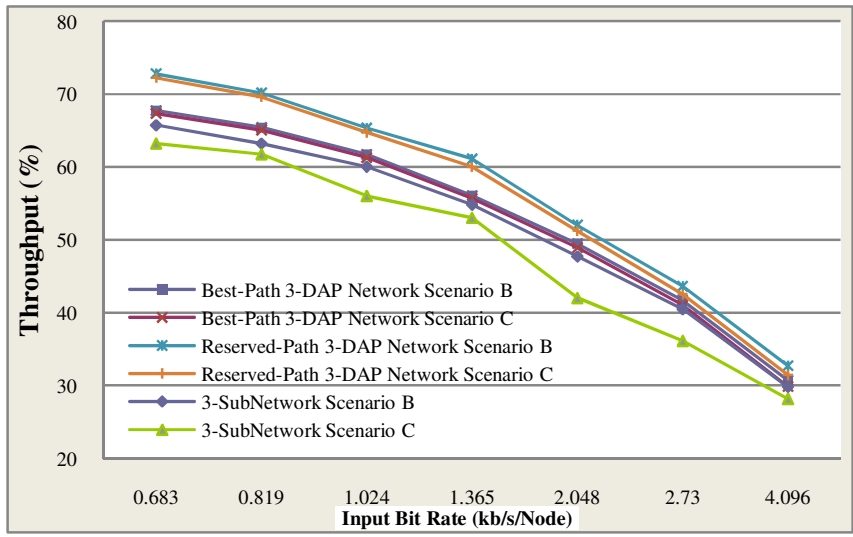

Fig. 7: The throughput performance of the Best-Path, Reserved-Path 3DAP Network and the 3-subnetwork schemes in Scenario B and Scenario $\mathrm{C}$ with 36 asymmetrical distributed meters.

based reserve-path scheme. Note that in the best-path scheme, the source node will select a DAP with the best route in order to send its data packets. The airtime link metric [7] is used to 
select the best route. In the timer-based Reserved-Path scheme meters will select the first best path to send a data packet, while keeping keep a copy of the data packet in its backup HWMP buffer. As soon as a link breakage notification is received for this path, the source node will send the packet through the second route, while it updates its tree table to the first root (DAP). Bear in mind that in both methods on-demand routing is used when a node experiences a link failure. From Fig. 5 we can observe a notable improvement in performance of the bestpath approach compared with the 3-subnetwork in scenario A. This is mainly because in scenario B, the border nodes will have an option to select the better path to one of the neighboring DAPs. In addition, the reserve-path scheme performs better than the best-Path scheme by attaining retransmission diversity.

Furthermore, we evaluate the self-healing aspect of the network in situations where some nodes (meters) or gateways malfunction. Fig. 6 shows the throughput performance of scenario A, as well as scenario B with the best-path and the timer-based reserved-path (airtime-link-based) under the following cases: Case 1 when all the meters work properly, Case 2 when 9 meters ( 3 meters in each region with more than two hops away from each DAP) stop functioning as soon as the simulation time reaches the half way point. From this figure it can be clearly observed that the flexibility of the best-path and the timer-based reserved-path schemes combined with the multipath feature of the multigate network structure can effectively allow packets to be re-routed by bypassing the malfunction nodes.

Finally, we compare the performance of the best-path, the timer-based reserved-path and the 3 sub-network schemes in scenarios $\mathrm{B}$ and $\mathrm{C}$ in Fig. 7, while in scenario $\mathrm{C}$ a higher number of meters (more traffic) are located in the vicinity of DAP-1. In Fig. 7 we also included the results of 3-subnetwroks under asymmetrical node distribution. The results in Fig. 7 confirm that the best-path and the reserved-path performance remains unaffected by asymmetrically distributed nodes, whereas in the case of 3 sub-network the throughput performance could suffer.

\section{CONCLUSION}

One of the most important challenges in smart grid is providing reliable last mile network communication. In this paper we propose a wireless mesh routing scheme with the ability to provide reliable communication from meters to the AMI headend via multiple gateways. In this multigate network structure we consider a tree-based routing scheme that is capable of providing every node with a separate path to each of the gateways. In addition, this multigate/multipath routing scheme is further exploited to provide a timber-based reservepath diversity routing. The performance of this network is then compared with a regional mesh network system where nodes in each region can only access their local gateway. We have shown that multigate routing can indeed enhance the reliability of the network. In addition, we have shown the scheme can enhance the self-healing ability of the network, such as handling the effect of malfunction nodes.

\section{REFERENCES}

[1] NIST Framework and Roadmap for Smart Grid Interoperability Standards, Release 1.0, Office of the National Coordinator for Smart Grid Interoperability, http://www.nist.gov/public_affairs/releases/upload /smartgrid_interoperability_final.pdf

[2] J. Anatory, N. Theethayi, R. Thottappillil, M. M. Kissaka and N. H. Mvungi, "The effects of Interconnections and Branched Network in the Broadband Powerline Communications", International Gathering of Radio Science, India, 23rd -29th October, 2005

[3] S. Galli and O. Logvinov, "Recent development in the standrdization of powerline communications within the IEEE”, IEEE Commun. Mag., 46 (7), pp. 64-71, July 2008.

[4] CENELEC, European Standard EN50065: "Signalling on low-voltage electrical installations in the frequency range $3 \mathrm{kHz}$ to $148.5 \mathrm{kHz}$ ", 2001.

[5] IEEE 802.11 Standard Working Group, Standard for Information Technology - Telecommunications and Information Exchange Between Systems - LAN/MAN Specific requirements Part 11: Wireless LAN Medium Access Control (MAC) and Physical Layer (PHY) Specifications, ANSI/ IEEE Std 802.11, first ed., 1999.

[6] H. Gharavi, K. Ban, "Multihop Sensor Network Design for Wideband Communications," THE PROCEEDINGS OF THE IEEE, vol. 91, NO. 8, August 2003, pp. 1221-1234.

[7] IEEE $802.11 \mathrm{~s}$ Task Group, Draft Amendment to Standard for Information Technology - Telecommunications and Information Exchange Between Systems - LAN/MAN Specific Requirements - Part 11: Wireless Medium Access Control (MAC) and physical layer (PHY) specifications: Amendment: ESS Mesh Networking, IEEE P802.11s/D1.0, November 2006.

[8] E. Royer and C. Perkins, " Multicast operation of the ad-hoc on-demand distance vector routing protocol", Proceedings of the ACM Mobicom '99, pages 207-218, August 1999.

[9] P. Sinha, R. Sivakumar, and V. Bharghavan, "MCEDAR: Multicast core extraction distributed ad-hoc routing", In Proc. of the Wireless Communications and Networking Conference, pp. 1313-1317, vol. 3, 1999.

[10] J. Zheng and M. J. Lee, "A Comprehensive Performance Study of IEEE802.15.4”, IEEE WPAN press 2006.

[11] C. Perkins, E. Belding-Royer, S. Das, Ad hoc on-demand distance vector (AODV) routing, IETF RFC 3561, 2003.

[12] Johnson, D. B., Maltz, D. A., and Hu, Y.-C. The Dynamic Source Routing Protocol for Mobile Ad Hoc Networks (DSR). IETF Internet Draft, draft-ietf-manet-dsr-10.txt, July

[13] Tsirigos and Z.J. Haas, "Multipath routing in the presence of frequent topological changes", IEEE Communications Magazine, Volume 39 Issue 11, Nov. 2001, p 132-138.

[14] S. Motegi and H. Horiuchi, "AODV-based multipath routing protocol for mobile ad hoc networks", IEICE Transactions on Communications, vol. E87-B, NO. 9, September 2004, pp. 2477-2483.

[15] S.K. Das, A. Mukherjee, S. Bandyopadhyay, K. Paul and D. Saha, "Improving quality-of-service in ad hoc wireless networks with adaptive multi-path routing", Proceedings of IEEE Global Telecommunications Conference (GLOBECOM '00), Volume 1, 27 Nov.-1 Dec. 2000, p 261265.

[16] S.J. Lee and M. Gerla, "Split multipath routing with maximally disjoint paths in Ad hoc networks", IEEE International Conference on Communications, v10, 2001, p 3201-3205.

[17] B. Yan and H. Gharavi, "Multi-path Multi-Channel Routing Protocol," IEEE International Symposium on Network Computing and Applications (IEEE NCA06), July 2006, Cambridge, MA USA.

[18] H. Gharavi, "Multichannel Mobile Ad Hoc Links for Multimedia Communications", Proceedings of the IEEE, vol. 96, No. 1, January 2008, pp. 77-96 